\title{
Les élections à Timor-Est, 2001-2012 : la démocratie au risque des tensions
}

\section{Stéphane Dovert et Frédéric Durand}

\section{(2) OpenEdition}

Journals

Édition électronique

URL : http://journals.openedition.org/echogeo/13241

DOl : 10.4000/echogeo.13241

ISSN : 1963-1197

Éditeur

Pôle de recherche pour l'organisation et la diffusion de l'information géographique (CNRS UMR 8586)

\section{Référence électronique}

Stéphane Dovert et Frédéric Durand, «Les élections à Timor-Est, 2001-2012 : la démocratie au risque des tensions », EchoGéo [En ligne], Sur le Vif, mis en ligne le 20 novembre 2012, consulté le 01 mai 2019. URL : http://journals.openedition.org/echogeo/13241 ; DOI : 10.4000/echogeo.13241

Ce document a été généré automatiquement le 1 mai 2019.

\section{(c) (i) (9)}

EchoGéo est mis à disposition selon les termes de la licence Creative Commons Attribution - Pas d'Utilisation Commerciale - Pas de Modification 4.0 International 


\title{
Les élections à Timor-Est, 2001-2012 : la démocratie au risque des tensions
}

\author{
Stéphane Dovert et Frédéric Durand
}

1 Localisée entre l'Indonésie et l'Australie, la République Démocratique de Timor-Leste, qui compte environ 1,2 million d'habitants sur $14900 \mathrm{Km}^{2}$, est une ancienne colonie portugaise d'Asie aussi connue sous le nom de Timor-Est ou Timor oriental (Cf. carte 1). Elle a été envahie puis occupée par l'Indonésie en décembre 1975 (Defert, 1992), avant d'accéder tardivement à l'indépendance après un référendum d'autodétermination mené sous l'égide de l'ONU le 30 août 1999 (Gunn, 1999; Dovert, 2001). Après un mois de troubles dus à une politique de terre brûlée menée par une partie de l'armée indonésienne, le territoire a été placé sous tutelle de l'ONU au début du mois d'octobre 1999, avant une pleine indépendance le 20 mai 2002.

2 Économiquement parlant, Timor-Leste, dont la viabilité avait été souvent mise en doute, et qui est encore fréquemment présenté comme le pays le plus pauvre d'Asie, réussit paradoxalement plutôt bien. De fait, il y a eu de réels progrès depuis la fin de la période d'occupation indonésienne. Ainsi, depuis 1999, l'espérance de vie est passée de 56 à 62,5 ans, tandis que le taux d'alphabétisation montait de 40 à $58 \%$, et le PIB par habitant de 330 à 5300 dollars (Durand, 2011). Grâce à des réserves d'hydrocarbures offshore, le gouvernement a en outre pu constituer un fonds de réserve qui s'élève en 2012 à plus de dix milliards de dollars, et qui lui permet une indépendance financière au moins pour les prochaines décennies.

3 En 2012, le pays a fêté dix années d'indépendance, parallèlement à l'organisation de ses troisièmes élections présidentielles et législatives. En mars 2012, la population de TimorLeste a choisi José Maria de Vasconcelos (de son nom de guerre: Taur Matan Ruak), soutenu par le Premier ministre sortant Xanana Gusmão, pour devenir le nouveau Président de la République. Quatre mois plus tard, les Timorais ont de nouveau voté le 7 juillet 2012, cette fois pour élire un nouveau Parlement composé de 65 députés pour une période de cinq ans. 
Les résultats marquent une relative continuité par rapport à ceux de 2007 et devraient conforter l'action du Premier ministre Xanana Gusmão, qui a été reconduit pour un nouveau mandat. Pour autant les événements qui ont jalonné ces élections montrent en même temps que l'essor de la démocratie « à l'occidentale » peut être source de tensions. Les processus électoraux sont en effet ici marqués par une histoire particulière qui, non sans paradoxe, leur donne un poids tout à fait singulier - Timor Leste est un des rares pays au monde à avoir acquis son indépendance grâce à un processus électoral -, dans un contexte où les élections sur le modèle occidental s'avèrent une pratique récente et où leurs attendus continuent à poser question.

Carte 1 - Timor Leste dans la région Asie-Pacifique

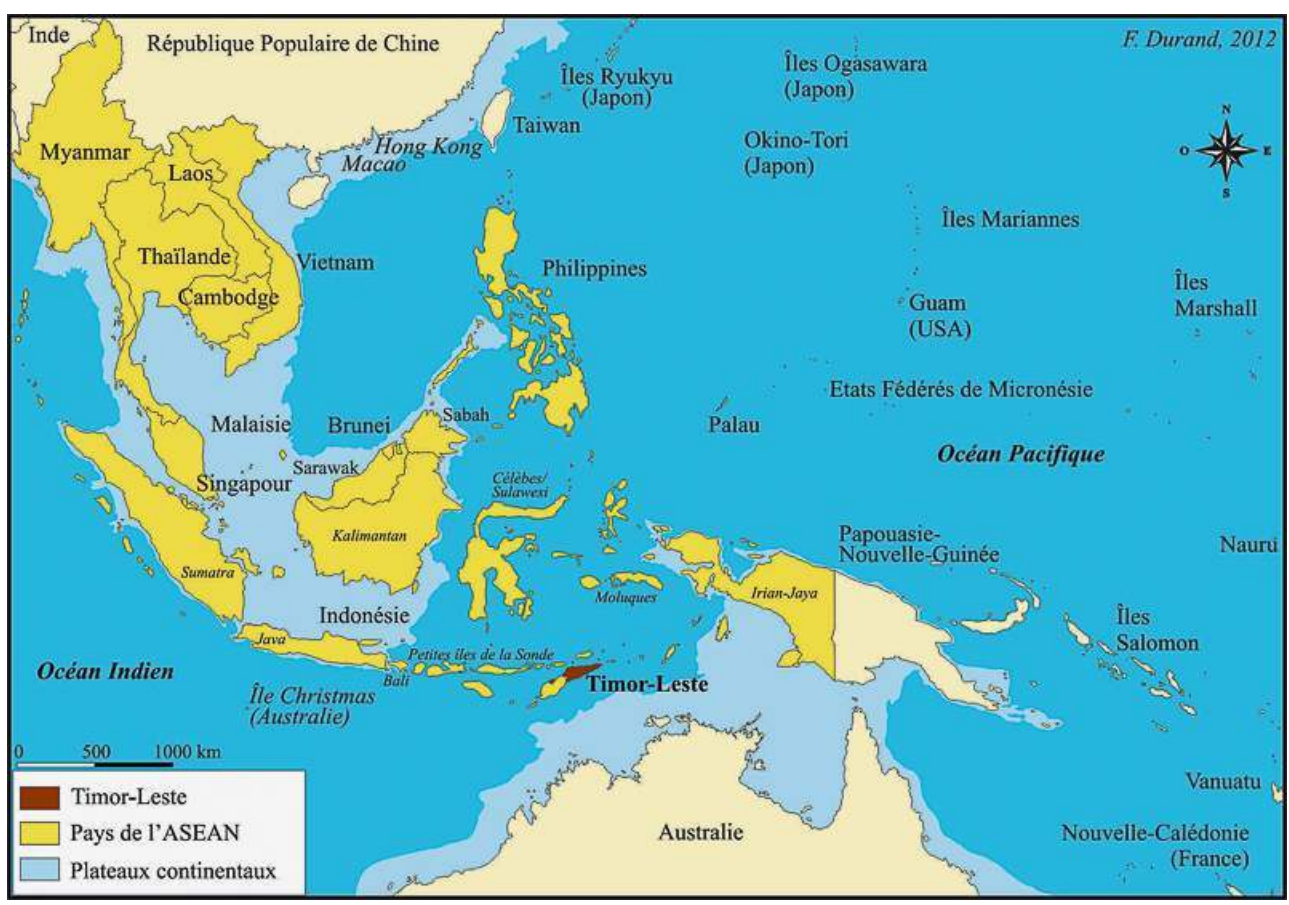

\section{Une tradition ancienne de « Républiques aristocratiques » face à la modernité politique}

5 On notera d'abord que dans la tradition politique occidentale, la notion de « démocratie » est fondamentalement attachée à celle d' «élection » qui en constitue en quelque sorte le premier symbole. Faute de processus électoral, on ne saurait, dans les " pays du Nord", qualifier un système de "démocratique». On se focalise ainsi sur les modalités d'accession au pouvoir bien davantage que sur les conditions de son exercice. Mais en sacralisant le système du suffrage universel dont l'expression répond à des critères effectivement forgés en Europe au cours des deux derniers siècles, soit l'expression d'un vote individuel, à bulletin secret sur la base d'une liste de candidature prédéfinies qui s'exclut les unes des autres, on s'interdit de prendre en considération d'autres traditions participatives qui, elles aussi, ont été élaborées dans l'idée du respect de l'expression collective.

6 Ainsi, par exemple, dans le territoire de l'actuel Afghanistan, l'affaiblissement des empires iranien et moghol au milieu du XVII ${ }^{\mathrm{e}}$ siècle, avait permis aux tribus afghanes de 
se débarrasser du joug extérieur et de se doter d'une structure de concertation: la Loya Jirga (Conseil des chefs tribaux). Cette structure a continué à fonctionner après l'indépendance du pays en 1919, même si son rôle s'est très sensiblement affaibli après la prise de contrôle soviétique, puis avec l'essor du régime taliban jusqu'en 2001. Après cette date, pour tenter de recréer l'unité de l'Afghanistan après ces déchirements, les négociateurs qui se sont penchés sur l'avenir du pays n'ont toutefois pas trouvé de meilleur moyen que de réactiver la Loya Jirga.

7 Au Timor Oriental, même si, au cours du troisième quart du $\mathrm{XX}^{\mathrm{e}}$ siècle, le régime salazariste aimait présenter le pays comme un ensemble d'ex-royaumes autocratiques, les modalités d'expressions populaires n'étaient pas exclues de la vie politique locale. Ainsi les souverains des multiples royaumes devaient-ils se soumettre aux décisions d'assemblées sur lesquelles leur pouvoir ne s'exerçait pas. Le gouverneur portugais Affonso de Castro qui s'est penché sur la question au milieu du XIX ${ }^{e}$ siècle, avait même qualifié les royaumes traditionnels timorais de «Républiques aristocratiques» (Castro, 1867). Dans ces proto-républiques, le roi était désigné par une assemblée de notables et ne pouvait prendre de décisions contraires à la volonté de la majorité de ses membres. Un souverain qui l'aurait fait aurait risqué d'être démis de ses fonctions et remplacé par un dirigeant plus enclin à suivre la volonté de la communauté. On signalera en outre qu'il y avait en réalité deux souverains, un pour les affaires intérieures (considéré comme « féminin» et garant de la stabilité), l'autre pour les affaires extérieures (qualifié de «masculin » et de moindre importance). Les notions de « "féminin » et de «masculin » doivent toutefois être prises au sens figuré ou symbolique, car il arrivait que des femmes assurent les activités extérieures et inversement.

Paradoxalement, c'est l'impact de la colonisation occidentale qui a renforcé le pouvoir autocratique des rois timorais. Le gouverneur portugais, pour exercer son autorité, voulait pouvoir s'adresser à un interlocuteur unique, et de préférence à un homme. Les nominations de reines sont devenues de plus en plus rares au début du XXe siècle, sachant que quand cela survenait, le gouverneur demandait la nomination d'un régent. La tradition de concertation s'est également estompée progressivement. Cette concertation est néanmoins restée active au niveau des villages. La fin de la période coloniale a ainsi été marquée par une dualité entre les différentes échelles du pouvoir. Le pouvoir colonial s'exprimait à Dili selon un mode radicalement autocratique et centralisé s'appuyant, pour exercer son mandat en province, sur une aristocratie traditionnelle soumise. En revanche, au niveau micro-locale, les schémas participatifs, peut-être hérités de la tradition mélanésienne (Defert, 1996) étaient peu altérés. Toute analyse de la forme d' «accélération de l'histoire » qu'a connue le Timor oriental au cours du dernier demisiècle doit tenir compte de ce substrat.

9 En 1974, la Révolution des ๔illets a fondamentalement modifié la donne politique au Portugal. Dans la colonie éloignée que représente alors Timor, un débat politique jusqu'à présent interdit par la dictature salazariste a pu émerger. Fort logiquement, il a été dans un premier temps animé au premier chef par les petites élites urbaines formées à l'occidentale et variablement influencées par les doctrines chrétiennes et marxiste (Defert, 1992). Deux partis politiques se sont formés dès le printemps 1974: l'ASDT (Association sociale démocrate Timoraise), qui allait se transformer par la suite en Fretilin (Front Révolutionnaire pour l'Indépendance de Timor-Est) et l'UDT (Union Démocratique Timoraise). Le premier était de sympathie communiste, à l'instar de son modèle mozambicain, le Frelimo avec lequel certains de ses jeunes leaders avaient été en 
contact lorsqu'ils faisaient leurs études à Lisbonne. Le deuxième était plus conservateur et plus lié aux élites anciennes. Il souhaitait une indépendance à moyen terme assortie du maintien de relations avec le Portugal.

10 La rivalité entre les deux partis allait à l'encontre des consensus traditionnels, en opposant notamment les "hommes du peuple» (maubere) aux "notables» et les « jeunes» aux « anciens ». La coalition établie en janvier 1975 entre le Fretilin et l'UDT s'inscrivait bien davantage dans l'esprit traditionnel du consensus. Elle n'a cependant pas résisté à la pression des services spéciaux indonésiens (Bakin) qui, en arguant qu'ils n'accepteraient pas de communistes dans un gouvernement est-timorais indépendant, ont convaincu l'UDT de tenter une prise de pouvoir par la force en août 1975 (Hill, 1978 ; Defert, 1992). Ces crises ont servi de prétexte à l'armée indonésienne pour envahir le territoire en décembre 1975.

11 Après des années d'un conflit très meurtrier mettant aux prises l'armée d'occupation à la population timoraise, les leaders indépendantistes ont à nouveau créé un front commun dans l'espoir d'obtenir un droit à l'autodétermination. C'est ainsi que le leader de la résistance, Xanana Gusmão, a proposé en 1988 la création d'un CNRM (Conseil National de la Résistance Maubere), qui s'est transformé ensuite en 1998 en CNRT (Conseil National de la Résistance Timoraise). Ce CNRT, qui constituait d'une certaine manière le prolongement de la coalition Fretilin-UDT, a cette fois également réuni plusieurs petits partis, dont l'ex-Apodeti qui s'était déclaré favorable à l'intégration à l'Indonésie en 1974 (Defert, 1992 ; Durand, 2002).

12 L'indépendance, obtenue définitivement au mois de mai 2012, doit assurément beaucoup aux évolutions de la vie politique en Indonésie et à la décision surprise du nouveau président B. J. Habibie qui, en 1998, a ouvert aux Timorais la possibilité d'une «consultation populaire» sous l'égide de l'ONU, l'expression «référendum d'autodétermination » étant proscrite pour ne pas faire perdre la face à l'Archipel. Nul doute cependant également que l'unité de la résistance, symbolisée par le Prix Nobel de la Paix conjointement attribué au leader indépendantiste José Ramos Horta et à l'évêque de Dili, Carlos Filipe Ximenes Belo en 1996, a considérablement joué en faveur de l'indépendance.

\section{1-2002 : les débuts victorieux du Fretilin, le parti de l'indépendance}

L'indépendance de Timor-Est s'est en quelque sorte forgée dans un consensus qui doit beaucoup aux valeurs traditionnelles, mais c'est un système référendaire importé qui a consacré le processus. Dès lors, une forme de «modernité démocratique » s'est s'imposée, éminemment légitime car elle a permis de tourner la page de l'occupation indonésienne, assurément la plus sombre de toute l'histoire timoraise, mais également porteuse de tensions et d'incompréhensions.

14 La «consultation populaire » a été organisée sous l'égide de l'ONU le 30 août 1999. Son résultat a été sans appel, plus de $97 \%$ de la population se rendant aux urnes pour voter à $78,5 \%$ pour l'indépendance. Après un mois de troubles liés aux exactions de l'armée indonésienne et des milices qu'elle avait créées, Timor-Est a été dirigé par une administration transitoire établie par l'ONU : l'UNTAET, qui a assuré la tutelle du territoire entre octobre 1999 et mai 2002. Dans un premier temps, les partis politiques de 
la fin de la période portugaise (1974/1975) se sont maintenus à l'intérieur du CNRT (Conseil National de la Résistance Timoraise), la plateforme commune de 1998.

Pendant la transition onusienne, un certain nombre d'observateurs avaient considéré que la meilleure option pour le début de l'indépendance aurait été un gouvernement d'union nationale, maintenant en place cet ancien CNRT. Cela aurait pu permettre de dépasser les clivages et amener la classe politique, numériquement peu importante, à chercher le consensus sur la meilleure manière de gérer le pays, en conformité avec les modes de gestion traditionnels est-timorais. On notera que l'actuel parti de Xanana Gusmão, également nommé CNRT mais sous une déclinaison différente (Conseil National pour la Reconstruction de Timor), et dont il sera question à partir de 2007, se distingue de son devancier en étant un parti politique spécifique et non une plateforme.

Malgré ses appels au rassemblement, fort de son aura de parti ayant piloté le combat pour l'indépendance, le Fretilin dirigé par Mari Alkatiri avait préféré s'engager dans la voie du multipartisme, comme en 1974/1975. Il pensait, non sans raison, avoir plus de chances que ses rivaux, et notamment que l'UDT, dont les dirigeants avaient dû se compromettre un temps avec le régime d'occupation indonésien. Xanana Gusmão avait donc dissout l'ancien CNRT afin de permettre l'organisation des élections d'août 2001, sans créer luimême de parti, car il comptait se présenter aux élections présidentielles d'avril 2002.

En août 2001, le Fretilin avait largement remporté les élections de l'Assemblée Constituante avec $57,4 \%$ des suffrages, loin devant le PD (Parti démocratique), qui avait obtenu 8,7\%, suivi de deux autres partis le PSD (Parti Social-Démocrate) et l'ASDT (Association Sociale Démocrate Timoraise) (Durand, 2002 - cf. tableau 1). De son côté, l'UDT qui avait collaboré avec le gouvernement indonésien pendant l'occupation, n'avait obtenu que $2,4 \%$ des suffrages et n'a jamais vraiment réussi à se relever depuis.

Tableau 1 - Les scores des principaux partis est-timorais aux élections législatives 2001-2012 (en \%)

\begin{tabular}{|l|l|l|l|}
\hline & 2001-2002 & 2007 & 2012 \\
\hline CNRT & - & 24,1 & 36,7 \\
\hline Fretilin & 57,4 & 29,0 & 29,9 \\
\hline PD & 8,7 & 11,3 & 10,3 \\
\hline Frenti-mudança & - & - & 3,1 \\
\hline PST & & 1,0 & 2,4 \\
\hline PSD & & & 2,1 \\
\cline { 1 - 4 } ASDT & 8,2 & 15,7 & \multirow{2}{*}{1,8} \\
\hline
\end{tabular}

Sources : Durand F., 2002 ; Cabasset C. et Durand F., 2007 ; Comissão Nacional de Eleições TimorLeste 2012.

Le fonctionnement de cette première Assemblée constituante n'avait pas été sans susciter des débats, notamment par rapport aux modalités de la rédaction de la Constitution. 
Xanana Gusmão avait souhaité que l'ensemble de la population soit consultée via une grande enquête, afin de déterminer les vraies priorités du peuple. L'Assemblée avait argué que cela serait trop coûteux et difficile à réaliser. Sur les conseils d'experts occidentaux, ses membres s'étaient contentés de modifier à la marge la Constitution du Portugal, en dépit du fait que les deux pays avaient des caractéristiques bien différentes.

Pour avoir contesté une décision de la majorité, Xanana Gusmão a été taxé par beaucoup d'hommes politiques locaux de penchants "anti-démocratiques ». Cet évènement est particulièrement intéressant, en ce qu'il pose la question de l'acception que l'on retient du concept même de démocratie. Dans le système timorais comme ailleurs, la question de la représentativité de l'« élu » et, subséquemment, celle du pouvoir qu'il convient ou non de lui déléguer est en jeu. En proposant une consultation populaire sur un texte fondateur pour son avenir, Xanana Gusmão se montrait assurément particulièrement fidèle à l'esprit de la démocratie. Il posait en revanche implicitement la question des limites de la délégation d'une décision politique majeure à une autorité distante.

La popularité de Xanana Gusmão, par affinité très enclin à se déplacer dans les villages distants où il a vécu ses années de résistance, est en tout état de cause restée très élevée. Il a été facilement élu Président de la République en avril 2002, devant Francisco Xavier do Amaral, proclamateur d'une première indépendance en novembre 1975 (Niner, 2010). ${ }^{1}$

21 À ce stade, au moment de l'accession à l'indépendance de mai 2002 plutôt que d'organiser de nouvelles élections, l'UNTAET avait préféré proposer la transformation de l'Assemblée constituante élue le 30 août 2001 en premier Parlement national de 2002 (Durand, 2001). Cette décision avait été contestée, certains objectant à juste titre que cela n'avait pas été expliqué clairement au départ, mais le pragmatisme avait prévalu, notamment pour des questions de coût.

Le score obtenu en 2001 avait permis à Mari Alkatiri, le Secrétaire général du Fretilin, de constituer le premier gouvernement national en mai 2002, en ayant une confortable majorité au Parlement avec 45 des 75 députés, les onze autres partis étant marginalisés. De fait, leurs voix étaient morcelées et le principal parti d'opposition, le PD, ne disposait que de 7 députés.

Le tandem Xanana Gusmão/Mari Alkatiri, dans le cadre de cette première législature, n'avait cependant pas très bien fonctionné. La Constitution nationale, largement inspirée du modèle portugais, donne un grand pouvoir exécutif au gouvernement issu de la majorité parlementaire, tandis que le Président assure surtout un rôle de garant de la Constitution et des institutions. Paradoxalement, ce placage du système occidental n'était pas forcément en opposition aux vraies pratiques anciennes de la démocratie timoraise, au contraire. En effet, la colonisation avait imposée l'image d'un pouvoir autocratique à un seul souverain, alors que la nouvelle Constitution de 2002, d'inspiration portugaise, permettait de restaurer le principe de deux pouvoirs, l'un garant de la stabilité (autrefois assuré par le «roi féminin» de l'intérieur), l'autre de l'action: le roi «masculin» de l'extérieur. Encore fallait-il que les deux personnalités en charge du pouvoir puissent s'accorder. Cela n'avait guère été le cas en 2002, d'autant que Xanana Gusmão aurait souhaité intervenir directement dans la gestion du pays, ce que ne lui permettait pas son statut de « Président ».

Dans ce contexte, nombre de ministres du Fretilin, qui avaient pour la plupart été peu préparés à l'exercice d'un tel pouvoir, n'ont pas su faire face à l'ampleur de la tâche. Le problème n'était pas tant le manque d'argent, que l'incapacité à l'utiliser. Ainsi, pour 
l'exercice budgétaire 2005/2006, à peine $62 \%$ des dépenses prévues avaient été effectuées. En outre, les ministres s'étaient souvent contentés de suivre les conseils des « experts» internationaux, qui poussaient généralement dans le sens d'un minimum d'intervention publique pour favoriser au maximum un secteur privé qui peinait à se former (Durand, 2008). Cette politique, qui peut paraitre paradoxale pour un parti parfois considéré comme "communiste", n'a pas permis de répondre aux attentes de la population. Les Timorais avaient en effet espéré que l'indépendance allait amener rapidement une amélioration sensible de leurs conditions de vie, et notamment l'octroi de pensions aux anciens combattants qui tardaient à être accordées.

En 2006, ce mécontentement s'était combiné avec un ralentissement de l'activité économique lié à un premier retrait massif mal préparé de l'ONU (dont la présence avait contribué jusqu'à $25 \%$ du PIB du territoire en 2001/2002), et avec une rébellion de pétitionnaires de l'armée se plaignant de discriminations, qui avaient pris les armes et refusé de retourner dans leurs casernes (Durand, 2008; Cabasset-Semedo \& Durand, 2009).

En 2006, ces troubles ont amené la destruction de milliers de maisons sur toute l'étendue du territoire et contraint près de 200000 personnes ( $20 \%$ de la population du pays) à se masser dans des camps de réfugiés internes. Le Président Xanana Gusmão a alors été obligé de demander le retour d'une force militaire multinationale pour restaurer l'ordre ainsi que la démission du Premier ministre Mari Alkatiri. Ce dernier a été remplacé pendant une phase d'intérim par José Ramos-Horta, qui bénéficiait d'une image très favorable comme leader de la résistance à l'extérieur. De fait, il avait représenté le territoire devant l'ONU pendant les années de lutte pour l'indépendance (1975-1999) et obtenu le Prix Nobel de la Paix en 1996, conjointement avec l'évêque de Dili, Mgr Carlos Filipe Ximenes Belo.

\section{7 : l'accession au pouvoir du nouveau CNRT}

L'année suivante, en 2007, les élections présidentielles et législatives nationales ont été placées sous le signe de la tension. Xanana Gusmão avait compris que le véritable pouvoir n'était pas aux mains du Président et il souhaitait intervenir plus directement dans la gestion du pays. Il créa donc son propre parti politique, en s'inspirant du sigle de l'ancienne plateforme de 1998-2001: le CNRT. Cette structure fût rebaptisée pour l'occasion: "Congrès National de Reconstruction de Timor ». Xanana Gusmão proposa ensuite à José Ramos-Horta de concourir pour la présidence, en se réservant lui-même pour les législatives.

28 Le premier tour de la présidentielle de 2007 avait placé en tête le candidat du Fretilin: Francisco Guterres, de son nom de guerre Lú-Olo, un ancien commandant de la guérilla contre l'Indonésie (tableau 2). José Ramos-Horta était cependant parvenu à rassembler davantage d'électeurs au deuxième tour et à obtenir une large majorité, devant son adversaire du Fretilin (Cabasset-Semedo \& Durand, 2007). Ces élections avaient aussi été marquées par la complexification de la vie politique timoraise, avec huit candidats se présentant à l'élection présidentielle, dont une femme : Lúcia Lobato, leader du PSD (Parti Social-Démocrate) devenue par la suite ministre de la Justice. 
Tableau 2 - Les scores au premier tour des élections présidentielles, 2002-2012 (en \%)

\begin{tabular}{|l|l|l|l|}
\hline & $\mathbf{2 0 0 2}$ & $\mathbf{2 0 0 7}$ & $\mathbf{2 0 1 2}$ \\
\hline Xanana Gusmão (CNRT) & 82,7 & - & - \\
\hline Francisco Xavier do Amaral & 17,3 & 14,4 & - \\
\hline José Ramos-Horta & - & 21,8 & 17,5 \\
\hline Francisco Guterres (Fretilin) & - & 27,9 & 28,8 \\
\hline Fernando Lasama (PD) & - & 19,2 & 17,3 \\
\hline Lúcia Lobato (PSD) & - & 8,9 & - \\
\hline José Maria de Vasconcelos & - & - & 25,7 \\
\hline
\end{tabular}

Sources : Durand F., 2002 ; Cabasset C. et Durand F., 2007 ; Comissão Nacional de Eleições TimorLeste 2012

Cette complexification de la vie politique s'est confirmée aux législatives de juillet 2007, avec quatorze partis en lice. Toutefois, une nouvelle règle empêchant les partis ayant obtenu moins de $3 \%$ d'obtenir un siège au Parlement en avait exclu la moitié. On signalera par ailleurs que sur les 65 députés, 21 étaient des femmes, soit $32 \%$, un des taux les plus élevés de la planète.

Un problème important a cependant surgi à l'issue de ces élections législatives de 2007, à propos de la désignation du Premier ministre. En effet, la Constitution nationale de 2002 recèle une ambiguïté dans sa formulation. L'article 106 stipule que « Le Premier ministre est désigné par le parti politique ou l'alliance de partis politiques ayant une majorité parlementaire, et est nommé par le Président de la République, après consultation des partis politiques siégeant au Parlement ».

31 De fait, le Fretilin pouvait être considéré comme le parti politique ayant « remporté » les élections et disposant d'une «majorité » des voix, dans la mesure où il avait obtenu $29 \%$ des suffrages contre $24 \%$ pour le CNRT de Xanana Gusmão. Mais le Fretilin connaissait en même temps l'usure de cinq années de pouvoir décevantes. En outre, les autres partis n'avaient pas souhaité former d'alliance avec le Fretilin, qui avait eu tendance à diriger sans véritable partage de 2002 à 2007.

Après un mois de concertations et de négociations extrêmement difficiles, le Président José Ramos-Horta s'est finalement résolu à demander à Xanana Gusmão de former un gouvernement, car contrairement au Fretilin, le CNRT était parvenu à s'allier avec les autres petits partis pour former une majorité parlementaire. Le Fretilin a argué du caractère inconstitutionnel de la décision, tandis que nombre de ses militants manifestaient avec violence, particulièrement dans l'est du pays. Conscient néanmoins de l'insuffisance de son socle parlementaire, le Fretilin s'est finalement résigné à entrer dans l'opposition.

33 Après 2007, la situation est restée tendue notamment en raison de la non-résolution de la rébellion militaire de mars-avril 2006. Une partie des pétitionnaires de l'armée se cachait 
toujours dans les montagnes et menaçait le pouvoir en place (Kingsbury \& Leach, 2007). Ce mouvement a toutefois trouvé un terme en février 2008, avec un double attentat manqué visant le Président de la République José Ramos-Horta et le Premier ministre Xanana Gusmão. Le Président a été grièvement blessé et a dû être hospitalisé en Australie, mais les leaders rebelles ont été tués ou se sont rendus aux autorités. Cela a mis fin à près de deux ans de crise et a permis au Premier ministre d'accélérer les réformes amorcées en 2007, notamment en ce qui concerne la construction d'infrastructures et le paiement de pensions aux anciens résistants comme aux plus démunis.

De fait, le pays, qui a longtemps été présenté comme un des "plus pauvres d'Asie", bénéficie en réalité de ressources importantes grâce aux hydrocarbures en mer de Timor, qu'il partage avec l'Australie et dont l'exploitation remonte aux années 1990. Cela lui assure des ressources d'environ un milliard de dollars par an, dont une majeure partie est placée sur un fonds sécurisé d'une banque aux États-Unis. Le fonds a dépassé les dix milliards de dollars en 2012 et sa gestion sur un modèle inspiré de la Norvège prévoit de garder une majeure partie du capital pour surtout utiliser les intérêts. Cette approche se justifie d'autant plus qu'en l'état actuel des explorations sismiques, ces ressources ne sont garanties que pour une quarantaine d'années. Cela laisse néanmoins une marge de manœuvre appréciable au pays, qui n'a quasiment plus besoin d'aide internationale, ni d'emprunter. Clin d'œil à l'histoire, la République Démocratique de Timor-Leste a même proposé en 2011, de conserve avec le Brésil, de racheter une partie de la dette souveraine du Portugal, son ancien colonisateur en difficulté financière.

\section{La confirmation du CNRT en 2012}

2012 s'annonçait comme une année particulièrement sensible pour Timor-Leste. D'un point de vue historique, le pays commémorait trois grands anniversaires : cinq cents ans de contacts avec les Portugais; un siècle depuis la fin de la dernière grande guerre coloniale, celle de Manufahi (1911-1912) et dix ans d'indépendance (Durand, 2009). Par ailleurs, l'essentiel de la représentation onusienne devrait quitter le pays à la fin de l'année, ce à quoi elle s'est mieux préparée qu'en 2006, en prenant des mesures pour limiter l'impact de son retrait, comme par exemple l'aide à la reconversion de ses anciens personnels locaux. Mais il restait un dernier test avant ce retrait : le bon déroulement des élections présidentielles et législatives de 2012 (Cabasset-Semedo, 2012). Le Fretilin s'était en effet beaucoup engagé dans la campagne afin de revenir au pouvoir dans le cadre de ces deux scrutins. Cela laissait augurer la possibilité d'une rupture ou de nouveaux désordres dans l'éventualité où le Fretilin obtiendrait comme en 2007 un meilleur score que le CNRT, sans pouvoir constituer de majorité.

Les élections présidentielles de mars 2012 ont réservé une relative surprise. Craignant sans doute une usure de l'image de José Ramos-Horta ainsi que l'aura de résistant armé du candidat Fretilin, Francisco Guterres dit Lú-Olo, le CNRT de Xanana Gusmão a préféré soutenir José Maria de Vasconcelos (dit Taur Matan Ruak), l'ex-commandant en chef de la résistance en 1999 (donc un ancien chef hiérarchique du candidat du Fretilin). Cette décision a écarté le Président sortant José Ramos-Horta dès le premier tour de la présidentielle. La stratégie s'est avérée payante car José Maria de Vasconcelos a remporté les élections avec près de $61 \%$ des suffrages devant Francisco Guterres (tableau 3). 
Tableau 3 - Les scores du deuxième tour des élections présidentielles, 2007 et 2012 (en \%)

\begin{tabular}{|l|l|l|}
\hline & $\mathbf{2 0 0 7}$ & $\mathbf{2 0 1 2}$ \\
\hline José Ramos-Horta & 69,2 & - \\
\hline Francisco Guterres (Fretilin) & 31,8 & 39,2 \\
\hline José Maria de Vasconcelos (CNRT) & - & 60,8 \\
\hline
\end{tabular}

Sources : Cabasset C. et Durand F., 2007 ; Comissão Nacional de Eleições Timor-Leste 2012.

Le premier tour des présidentielles avait cependant montré une certaine dispersion des voix avec quatre candidats obtenant plus de $17 \%$ des voix (Cf. tableau 2): les deux finalistes, mais aussi José Ramos-Horta et Fernando de Araújo (dit Lasama) le fondateur du Parti Démocratique (PD). Ce dernier représente la génération montante des personnalités politiques du pays. En effet, contrairement aux principaux autres leaders, Fernando de Araújo, qui a été Président du Parlement de 2007 à 2012, n'est pas issu de la génération de la fin de la période coloniale portugaise. Né en 1963, il s'est fait remarquer pendant l'occupation indonésienne en devenant le Secrétaire général du Renetil, le réseau de résistance des étudiants est-timorais. Cela lui a valu d'être arrêté et torturé en 1991, puis d'être condamné à six ans d'emprisonnement par un tribunal militaire indonésien.

Le Parti Démocratique aurait pu créer la surprise aux législatives de juillet 2012, car José Ramos-Horta, le Président sortant, lui avait apporté son soutien, sachant que si l'on additionnait leurs deux scores du premier tour de la présidentielle de mars 2012, on parvenait à près de $35 \%$, soit plus que le Fretilin ou le CNRT (Cf. tableau 2). Un certain éclatement des voix était également possible, car il y avait 21 partis en lice pour les législatives de juillet.

Les électeurs ont finalement préféré jouer la continuité, en faisant confiance à l'actuel Premier ministre, d'autant que les législatives se déroulaient sur un seul tour, et que José Ramos-Horta ne disposait pas d'un parti politique propre. Alors que le candidat du CNRT, le nouveau Président José Maria de Vasconcelos (Taur Matan Ruak), n'avait obtenu que $25,7 \%$ des suffrages au premier tour de la présidentielle (tableau 1), le CNRT a atteint $36,7 \%$ aux législatives. De son côté, le Fretilin a légèrement progressé $(29,9 \%$ contre $28,8 \%$ ), mais pas suffisamment pour arriver en tête.

Les autres partis en revanche ont subi un revers. Le PD de Fernando Lasama, qui avait fait $11,3 \%$ aux législatives de 2007 et 17,3\% au premier tour des présidentielles, n’a remporté que $10,3 \%$ des suffrages, en dépit du soutien de José Ramos-Horta. L'autre perdant des législatives est le Parti Social-Démocrate de Lúcia Lobato. En 2007, il s'était associé à l'ASDT du proclamateur de la République de 1975 : Francisco Xavier do Amaral. Cela avait permis à cette petite coalition d'obtenir 15,7 \% des votes. En juillet 2012, les deux partis se sont présentés séparément. Cela s'est révélé une piètre stratégie, d'autant que l'ASDT a pâti du décès de Francisco Xavier do Amaral. Ces deux partis n'ont obtenu respectivement que 2,1 et 1,8\% des voix, ce qui ne leur a pas permis d'avoir de député, en raison de la règle des $3 \%$ minimum.

41 Un nouveau parti en revanche a émergé, même si c'est modestement : le Frenti-mudança, ${ }^{2}$ de l'ancien vice-Premier ministre José Luís Guterres. Il s'agit d'une composante dissidente 
du Fretilin, qui s'est formée en 2011 et est relativement proche de l'actuel Premier ministre Xanana Gusmão. On signalera également la montée du PST, le Parti Socialiste Timorais, qui est passé de 1 à $2,4 \%$ des voix entre 2007 et 2012. Cela n'est pas suffisant pour lui donner un député, mais indique l'amorce d'une opposition politique sensiblement plus radicale et engagée que le Fretilin.

\section{Le poids de la géographie des votes}

La répartition géographique des votes aux législatives de 2012 est également intéressante pour mesurer l'adhésion des populations au système électoral «à l'occidentale » et les possibles effets pervers de celui-ci.

De fait, l'implantation régionale joue beaucoup dans les résultats des treize districts. Les présidentielles et les législatives de 2007 avaient montré ce phénomène. Le Fretilin était particulièrement bien implanté dans les trois districts de l'extrême-est du pays (Baucau, Viqueque, Lautém) où la résistance armée s'était concentrée durant l'occupation indonésienne. Le CNRT dominait les districts de la côte nord-ouest et du centre (Liquiçá, Dili, Manatuto), tandis que le Parti Démocratique s'imposait plus sur le sud-ouest (Ermera, Bobonaro, Cova Lima, Ainaro).

La répartition des votes aux législatives de 2012 confirme cette tendance, même si on peut constater une légère érosion du Fretilin dans le district de Lautém, à l'extrémité orientale du pays, où le CNRT et de PD ont fait tous les deux une percée à plus de $20 \%$ (cartes 2, 3 et 4).

Carte 2 - Résultats du CNRT aux élections législatives de juillet 2012

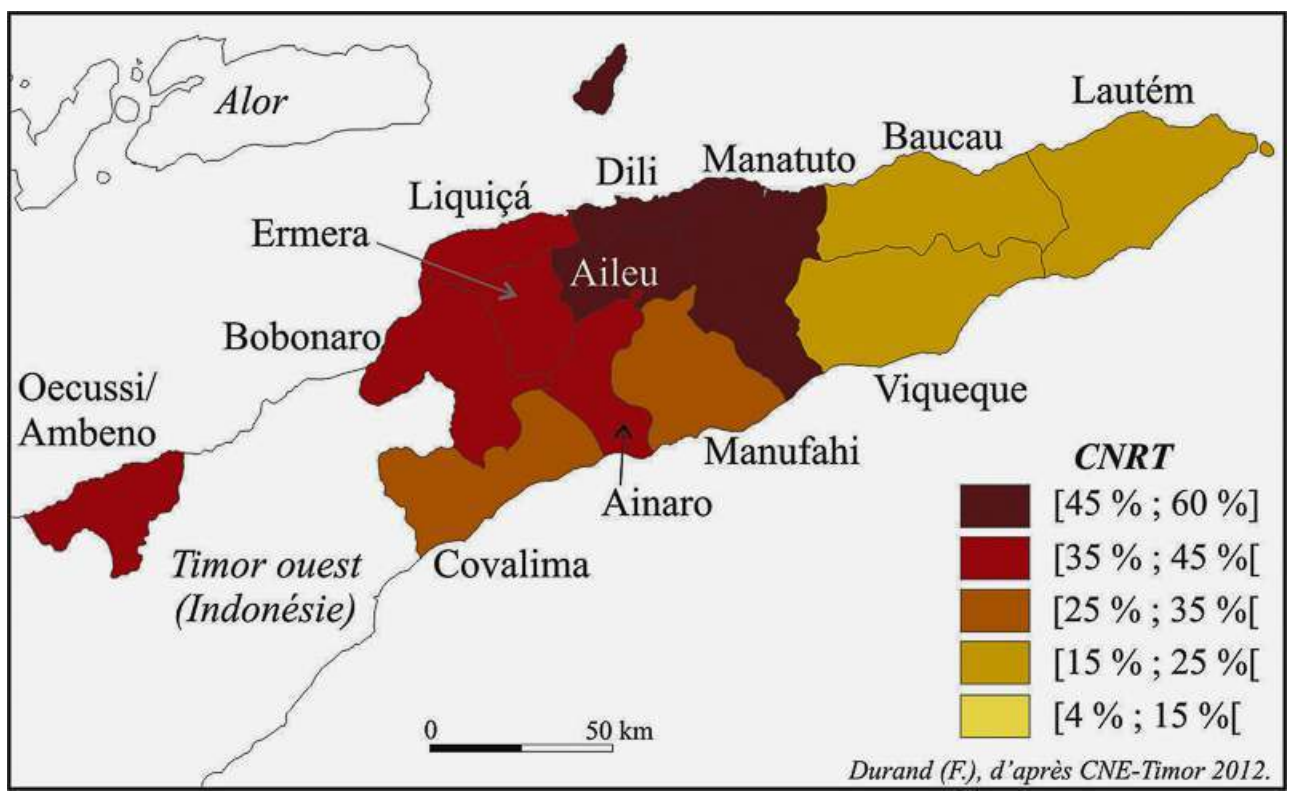


Carte 3 - Résultats du Fretilin aux élections législatives de juillet 2012

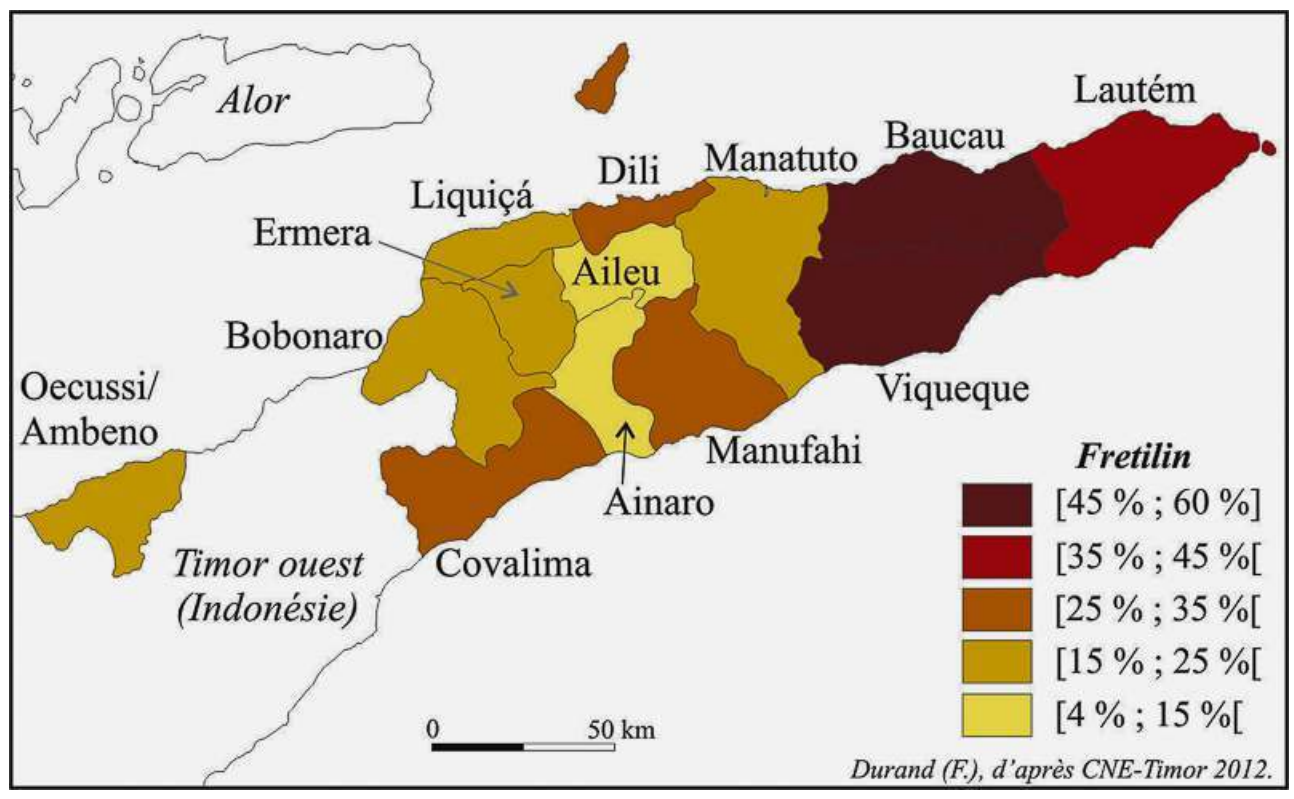

Carte 4 - Résultats du PD aux élections législatives de juillet 2012

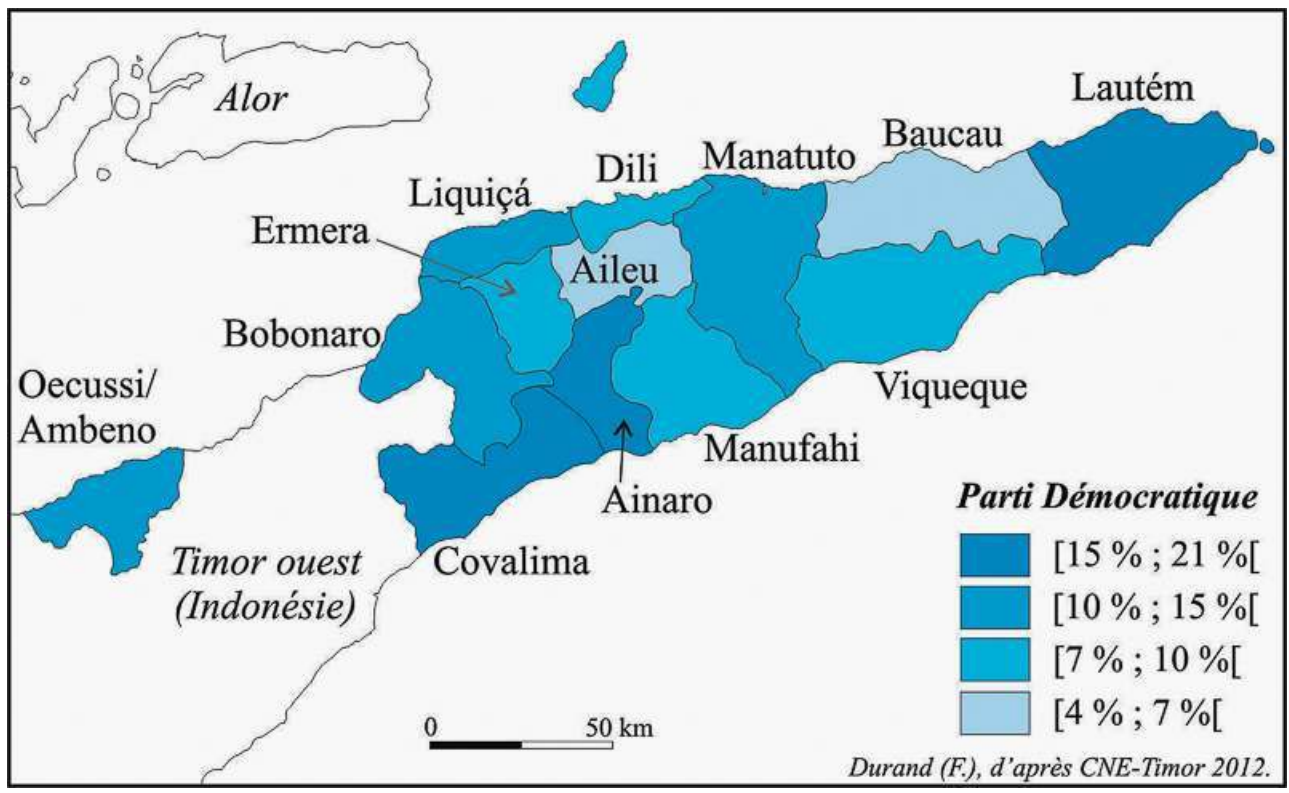

Le CNRT a renforcé son influence au centre du pays, particulièrement dans le district de la capitale Dili et à Aileu, où il gagne environ la moitié des suffrages. Sur ce dernier district, le CNRT a sans doute bénéficié du report des voix d'une partie des partisans de l'ASDT, le parti de Francisco Xavier do Amaral, le proclamateur de 1975, qui a perdu de son audience avec le décès de son chef en mars 2012.

Le Parti Démocratique conforte sa position de troisième parti national, sans vraiment parvenir à décoller puisque son meilleur score est d'à peine plus de $20 \%$ à Lautém. De son côté, le Frenti-mudança, parti dissident du Fretilin, n'est pas encore parvenu à s'installer solidement, oscillant entre 1,4 et $4,7 \%$ selon les districts, sauf dans l'enclave d'Oecusse, dans la partie occidentale de Timor, où il atteint $10,4 \%$. De fait, dans son ensemble, la 
population de ce district relativement isolé n'a jamais été vraiment affiliée à un parti particulier.

47 Les coïncidences entre fracture politique et fracture géographique ont été mises en exergue par le processus électoral qui les a assurément nourries et accentuées. La mosaïque ethnolinguistique timoraise est de toute évidence agitée par des élections qui exacerbent les antagonismes. Nul doute non plus que le principe même de la circonscription électorale conduise à renforcer les logiques de fief.

On mettait plus haut l'accent sur les traditions participatives au sein des villages timorais. S'ils sont historiquement avérés, il est difficile, faute de données suffisantes d'en percevoir les nuances géographiques, mais également d'en déterminer les contours exacts. Quelles catégories de personne étaient ainsi éligibles pour participer aux décisions engageant la communauté ? On sait que la séniorité avait beaucoup d'importance. En outre, le rôle des femmes dans la partie la plus orientale de l'île où les systèmes de dot sont les plus contraignants, n'était pas forcément le même qu'à l'ouest.

Le souci apporté à la représentation des femmes dans les processus électoraux récents s'est confirmé via un amendement à la loi électorale en juin 2011, qui oblige les listes proposées par les partis politiques à comprendre au moins un tiers de femmes. De ce fait, 21 femmes ont été élues en juillet 2012, soit $32 \%$ du Parlement. Ces chiffres restent néanmoins équivalents à ceux des législatives de 2007, menées antérieurement au système des quotas.

Le taux de participation mérite aussi d'être commenté (tableau 4). Ayant durement conquis le droit à l'indépendance après vingt-quatre ans de lutte, les Timorais de l'Est restent bien plus attachés à leur devoir électoral que la plupart des citoyens des pays du Nord. La multiplication des élections (dix en une douzaine d'années) a cependant eu tendance à provoquer une légère érosion de leur niveau de participation. À leur décharge, une partie des abstentionnistes a fait remarquer en 2012 que les procédures électorales ne permettaient pas pour l'instant les votes par procuration et qu'il était nécessaire de se rendre dans sa commune de recensement. De nombreux jeunes ayant migré en ville se sont ainsi plaints de ne pas avoir pu voter car ils ne disposaient pas du temps ou de l'argent pour effectuer le déplacement.

Tableau 4 - Taux de participation aux élections à Timor-Est 1999-2012 (en \%)

\begin{tabular}{|l|l|l|l|l|l|l|l|l|l|}
\hline Référ. & Constit. & Présid & Prés.1 & Prés.2 & Législ. & locales & Prés.1 & Prés.2 & Législ. \\
\hline $\mathbf{1 9 9 9}$ & $\mathbf{2 0 0 1}$ & $\mathbf{2 0 0 2}$ & $\mathbf{2 0 0 7}$ & $\mathbf{2 0 0 7}$ & $\mathbf{2 0 0 7}$ & $\mathbf{2 0 0 9}$ & $\mathbf{2 0 1 2}$ & $\mathbf{2 0 1 2}$ & $\mathbf{2 0 1 2}$ \\
\hline 98,6 & 94,6 & 86,3 & 81,8 & 78,8 & 80,5 & 67,7 & 78,2 & 73,1 & 74,8 \\
\hline
\end{tabular}

Successivement : référendum de 1999, élections de l'Assemblée constituante, présidentielle à un tour de 2002, les deux tours de la présidentielle de 2007, les législatives de juillet 2007, les élections des chefs de villages d'octobre 2009, les deux tours de la présidentielle de 2012 et les législatives de juillet 2012.

Sources : Durand F., 2001; Cabasset C. et Durand F., 2007 ; Durand F., 2008 ; Comissão Nacional de Eleições Timor-Leste 2012. 


\section{Les coalitions face à l'exercice de la démocratie}

51 Avec ces résultats en juillet 2012, le CNRT a significativement consolidé sa position même si, avec 31 sièges de députés, il n'est pas parvenu à obtenir la majorité absolue. Il lui aurait fallu en effet 33 sièges par rapport aux 65 de la législature (tableau 5).

Tableau 5 - Les scores et les députés au Parlement en juillet 2012

\begin{tabular}{|l|l|l|l|l|}
\hline & CNRT & Fretilin & PD & Frenti-mudança \\
\hline Pourcentage de votes & $36,7 \%$ & $29,9 \%$ & $10,3 \%$ & $3,1 \%$ \\
\hline Nombre de députés & 31 & 24 & 8 & 2 \\
\hline
\end{tabular}

Source : Comissão Nacional de Eleições Timor-Leste 2012

Cette situation a induit des tractations serrées, d'autant que pendant plusieurs jours, il y a eu des incertitudes sur le nombre exact de député du CNRT : 30 ou 31. Avec 31, le CNRT pouvait faire alliance avec n'importe lequel de ses trois rivaux pour obtenir une majorité absolue de 33 députés, même si une alliance avec le Frenti-mudança aurait été risquée, car ne laissant aucune marge de manœuvre. Avec 30, cela l'obligeait à s'allier forcément avec le PD ou le Fretilin. Le PD pouvait paraître le partenaire le plus évident, dans la mesure où il faisait partie de la précédente coalition avec le CNRT de 2007 à 2012. Mais tout en négociant, il a fait savoir, par son Secrétaire général qu'il n'était pas contre l'idée d'entrer dans l'opposition. À l'inverse, les membres du Fretilin, manifestement déçus de ne pas être parvenus à accroître leur base, se déclaraient prêts à travailler avec le CNRT et à entrer au gouvernement. Cette possibilité d'aller dans le sens de la coopération plutôt que de la confrontation a été vivement soutenue par l'ex-Président José Ramos-Horta et par l'évêque de Dili, Alberto Ricardo. De fait, l'Église, qui joue un rôle important dans ce pays catholique à plus de $95 \%$, assure depuis des années un rôle de garant des engagements de bonne conduite des partis politiques.

D'une certaine manière un rapprochement CNRT/Fretilin aurait constitué un retour à l'ancienne formule de plateforme politique commune de 1988 à 2001 (CNRM, puis ancien CNRT), que le Fretilin n'avait pas souhaité prolonger au moment des élections de l'Assemblée constituante. Mais en 2012, le Fretilin avait compris que rester encore cinq ans hors du gouvernement risquait de fragiliser encore plus sa position. L'option de la coalition n'avait cependant pas que des partisans, notamment parmi les ONG de la société civile, dont la très influente Lao'Hamutuk, qui craignaient qu'une majorité CNRT/Fretilin de 55 députés sur 65 n'étouffe toute opposition et donc le fonctionnement démocratique "à l'occidentale ». Ces orientations illustrent bien les hésitations que peuvent connaître les dirigeants des pays du Sud, entre le souhait d'avoir une majorité la plus large possible, quitte à accepter d'importants compromis, et l'option d'entrer dans un jeu tendant vers le monopartisme, au risque de troubles dans la rue.

Finalement, après une semaine d'attente, c'est l'option d'une coalition CNRT/PD/Frentimudança qui a été retenue, au détriment de la possibilité CNRT/Fretilin. Le rejet de cette dernière était prévisible, car le Fretilin avait notamment axé sa campagne sur la critique du plan de développement à l'horizon 2030, présenté par Xanana Gusmão à l'assemblée 
des bailleurs de fonds du pays en août 2011 pour prolonger les avancées réalisées après l'indépendance et surtout depuis 2007 (República Democrática de Timor-Leste, 2011). Le Fretilin avait assuré à ses électeurs qu'en cas de victoire, il reviendrait au plan de 2002, pourtant bien moins construit et cohérent. Par delà les problèmes d'ego entre Xanana Gusmão et Mari Alkatiri, cette divergence sur les plans stratégiques à mettre en œuvre s'est révélée relativement rédhibitoire, même si les critiques du Fretilin à propos du nouveau plan n'avaient pas forcément révélé de différences réelles majeures de conception. Le CNRT a toutefois commis l'erreur d'annoncer sa décision à l'issue d'un congrès qui avait été entièrement diffusé sur la télévision nationale, et où plusieurs membres du CNRT avaient critiqué le Fretilin de manière vive. La déception des électeurs du parti indépendantiste d'être écartés du gouvernement s'est donc doublée d'une impression d'avoir été publiquement dénigrés. Des manifestations violentes ont ainsi eu lieu pendant plusieurs jours, qui ont provoqué la destruction d'une soixantaine de voitures et le décès d'un jeune partisan du Fretilin.

Même si le contexte était sensiblement différent, ce moment de tension fait écho à celui de juillet 2007, lorsque le Fretilin n'avait pas accepté qu'on ne lui confie pas la formation du gouvernement.

\section{2-2017: un nouveau mandat et des défis pour confirmer la construction nationale}

La nouvelle coalition a obligé le CNRT à confier des postes clés aux autres composantes, qui auront vraisemblablement une influence significative sur les élections à venir. Ainsi, Fernando La Sama de Araújo, le dirigeant du PD, s'est vu confier le poste de Vice-premier ministre en charge des affaires sociales, tandis que José Luís Guterres, du Frenti-Mudança, obtenait le Ministère des Affaires étrangères.

Avec cette nouvelle configuration, le Premier ministre Xanana Gusmão dispose de conditions favorables pour continuer à redresser le pays et mettre en œuvre son plan de développement à l'horizon 2030. Cela étant, les améliorations constatées sont pour partie dues aux revenus des hydrocarbures, qui ne dureront pas toujours. En outre, la situation reste délicate, avec une croissance démographique non négligeable. La population, qui atteint 1,2 million d'habitants en 2012, pourrait s'élever à 2,5 voire près de 3 millions à l'horizon 2050. Cette croissance induit des enjeux majeurs en termes socio-économiques, que le plan de développement s'efforce d'anticiper. Il prévoit notamment de continuer une politique sociale de soutien aux plus démunis (près de 90000 personnes en 2011), de travailler à l'autosuffisance alimentaire et d'améliorer les infrastructures, particulièrement dans les secteurs du transport et de l'énergie. Le contrôle des naissances dans un pays profondément catholique reste un sujet aussi crucial que tabou.

Timor-Est va aussi être confronté à des choix lourds de conséquences pour l'avenir, comme par exemple de savoir s'il doit continuer le bras de fer avec la compagnie pétrolière australienne Woodside, qui bloque l'avancée de l'exploitation du grand gisement de Greater Sunrise en mer de Timor. En effet, l'Australie préfèrerait que la transformation du gaz se fasse sur son territoire ou sur une plateforme en mer, alors que les Timorais souhaitent que les infrastructures industrielles soient réalisées sur la côte sud de Timor, afin de créer une filière industrielle génératrice d'emplois. 
Un autre dossier complexe est celui de la gestion des revenus des royalties du pétrole et du gaz, pour l'instant placés sur un fonds avec de lourdes contraintes tant en matières de sécurité de placement que de montant mobilisable pour être versé au budget de l'État. Jusqu'en septembre 2011, l'argent était investi à $95 \%$ dans des placements garantis, principalement des bons du Trésor américain. Le montant des intérêts était réduit, mais garanti. De ce fait, le pays n'a pas souffert de la crise financière de 2008. Dorénavant, jusqu'à la moitié du fonds peut faire l'objet de placements plus risqués. D'autres débats sont aussi en cours sur l'ampleur des dépenses courantes par rapport à ce qui doit rester capitalisé sur le fonds, et sur les avantages et inconvénients d'emprunter de l'argent sur les marchés financiers pour alimenter le budget (qui atteint 1,7 milliard de dollars en 2012) plutôt que de ponctionner le fonds lui-même.

\section{Conclusion : quelles valeurs politiques pour la nouvelle nation timoraise}

Dans le contexte politico-financier mondial actuel, il n'y a sans doute pas de solution simple dans la gestion des affaires politico-économiques. Reste que, de manière presque paradoxale, après des décennies de discours mettant en doute sa viabilité, Timor-Leste dispose d'atouts non négligeables avec un territoire de taille non négligeable (une fois et demi le Liban), une population encore relativement limitée et des ressources en hydrocarbures au moins pour plusieurs décennies. Mais une erreur de stratégie pourrait être lourde de conséquences car la marge de manœuvre demeure limitée.

61 Du point de vue des relations internationale, la situation s'annonce plutôt positive. Après le succès de ces élections, la majorité des personnels de l'ONU devrait quitter le pays à la fin de l'année 2012. Par ailleurs, les relations avec l'Indonésie sont désormais apaisées, au prix du renoncement à un tribunal international pour juger les crimes contre l'humanité commis à Timor-Est entre 1975 et 1999. L'amélioration de ces relations devrait permettre au pays de devenir membre de l'Association des Nations d'Asie du Sud-Est (ASEAN) en 2013, et donc de renforcer son intégration régionale.

D'un point de vue électoral, par-delà les manifestations violentes de l'après législatives, toute la communauté internationale a salué la maturité politique du pays, y compris la Secrétaire d'État américaine Hilary Clinton, lors de sa visite à Timor le 6 septembre 2012. Il reste que, d'un point de vue sociétal, le sentiment d'appartenance politique des timorais de l'Est demeure beaucoup plus passionnel qu'en Occident, les défaites du Fretilin ayant notamment été vécues comme une injustice profonde par ses partisans, particulièrement dans l'extrême-est du territoire. On notera ici que ces clivages sensibles ne relèvent pas, et c'est là une différence notable avec l'Occident, de différences de situations socioprofessionnelles.

Ces confrontations sont apparues lors de la création des partis en 1974 et elles ont été d'une certaine manière réactivées à partir de 2001 par la volonté de promouvoir le multipartisme sur le modèle occidental, au détriment d'une plateforme commune. Pardelà l'avantage que cela peut générer en terme de pluralité d'expression, cette approcha va à l'encontre de l'aspiration traditionnelle à un consensus autour d'un chef fédérateur, surtout lorsque plusieurs pouvoirs forts apparaissent. Compte tenu de l'implantation géographique de l'électorat, même si l'érosion du Fretilin continue jusqu'aux prochaines 
élections de 2017 en raison de sa non-participation au gouvernement, il n'est pas sûr que le risque de tensions puisse disparaître rapidement, en tout cas à l'extrême-est du pays.

Plus globalement, par-delà l'urgence de la réalisation d'infrastructures et de la création d'emplois pour une population très jeune, la question du modèle de société voulu par la population n'a pas été élucidée. Lorsqu'ils sont interrogés, les Timorais de l'Est déclarent souvent ne pas vouloir que leur pays devienne à l'image de Singapour ou de Brunei (dont ils n'ont au demeurant qu'une image très distante), mais aspirer plutôt au maintien de leur culture et de ses spécificités. Les bases concrètes d'une telle réflexion n'ont toutefois jamais été clairement établies, notamment depuis que l'Assemblée constituante a renoncé à interroger la population en 2001, pour promulguer une Constitution largement calquée sur le modèle portugais. Les processus électoraux réguliers participent finalement de la même logique.

\section{BIBLIOGRAPHIE}

Cabasset-Semedo $C$., 2012. Timor-Leste. Le maintien de progrès et de la stabilité sur fond d'échéances électorales. In Jammes J. et de Tréglodé B. (dir.), Asie du Sud-Est 2012 : les événements majeurs de l'année, Bangkok-Paris, IRASEC-Les Indes savantes, p. 269-287.

Cabasset-Semedo C. et Durand F. (eds.), 2009. East-Timor, How to Build a New Country in Southeast Asia in the 21st Century. IRASEC (Institut de Recherche sur l'Asie du Sud-Est Contemporaine)- CASE (Centre Asie du Sud-Est), Bangkok-Paris, 299 p.

Cabasset-Semedo C. et Durand F, 2007. Les élections présidentielles de 2007 à Timor Leste. EchoGéo, mise en ligne le 16 juillet 2007.

Castro A., 1867. As Possessões Portuguezas na Oceania. Imprensa Nacional, Lisboa, xxi-460 p.

Defert G., 1996. L'Indonésie et la Nouvelle-Guinée-Occidentale. Maintien des frontières coloniales ou respect des identités communautaires. L'Harmattan, Paris, $454 \mathrm{p}$.

Defert G., 1992. Timor-Est, le génocide oublié: droit d'un peuple et raisons d'États. L'Harmattan, Paris, $323 \mathrm{p}$.

Dovert S., 2001. Timor Lorosa'e : un nouvel État à l'heure du village global - Réflexions sur nos mythes et nos modes. Lusotopie, p. 327-345.

Durand F., 2009. 42000 ans d'histoire de Timor-Est. Éditions Arkuiris, Toulouse, 130 p.

Durand F., 2008. Timor-Leste en quête de repères, perspectives économico-politiques et intégration régionale 1999-2050. Editions Arkuiris-IRASEC, Toulouse-Bangkok, 2008, 216 p.

Durand F., 2002. Timor Lorosa'e, Pays au carrefour de l'Asie et du Pacifique, un atlas géo-historique. Presses Universitaires de Marne-la-Vallée/IRASEC, Marne-la-Vallée/Bangkok, 208 p.

Durand F., 2001. Timor Lorosa'e 1930-2001 : partis politiques et processus électoraux à hauts risques. Aséanie, n8, p. 103-126.

Gunn (Geoffrey C.), 1999. Timor Loro Sae: 500 Years. Livros do Oriente, Macau, 323 p. 
Hill H. M., 1978. Fretilin: the Origins, Ideologies and Strategies of a Nationalist Movement in East Timor. M.A. Monash University, Australia, 236 p.

Kingsbury D. \& Leach M. (eds), 2007. East-Timor beyond Independence. Clayton, Monash University Press, xii-302 p.

Niner S., 2010. Xanana, Leader of the Struggle for Independent Timor-Leste. Australia Scholarly Publishing, $296 \mathrm{p}$.

República Democrática de Timor-Leste, 2011. Timor-Leste Strategic Development Plan 2011-2030. Dili, $231 \mathrm{p}$.

\section{NOTES}

1. Devant la menace imminente d'une invasion indonésienne (qui de fait devait avoir lieu dix jours plus tard), le Fretilin avait déclaré unilatéralement l'indépendance par rapport au Portugal le 28 novembre 1975. Francisco Xavier do Amaral, qui dirigeait alors le Fretilin, était devenu le premier Président de la République Démocratique de Timor-Leste, même si le pays n'avait pas été reconnu à l'époque par la majorité de la communauté internationale, notamment du fait du poids politique de l'Indonésie. Francisco Xavier do Amaral est décédé le 6 mars 2012 et n'a donc pas pu participer aux élections présidentielles du 17 mars 2012 auxquelles il s'était inscrit.

2. Littéralement: Frente de Reconstrução Nacional de Timor-Leste - Mudança «Front pour la Reconstruction National de Timor-Leste - Changement ».

\section{AUTEURS}

\section{STÉPHANE DOVERT}

Stéphane Dovert, Stephane.Dovert@diplomatie.gouv.fr, est membre associé de l'UMR 7306 IrAsia.

\section{FRÉDÉRIC DURAND}

Frédéric Durand, frederic.b.durand@gmail.com, est Maître de conférences à l'Université Toulouse II-Le Mirail et membre de l'UMR 7306 IrAsia. 